\title{
Risk of Thromboembolism in Patients Suffering from Multiple Sclerosis
}

\section{Arpaia $\mathbf{G}^{1 *}$, Bavera $\mathrm{P}^{2}$, Ippolito $E^{3}$, Spezzigu $\mathbf{G}^{1}$, Agus $\mathrm{GB}^{3}$ and Cimminiello $\mathrm{C}^{1}$}

${ }^{1}$ Vascular Medicine, Desio and Vimercate General Hospital, Italy

${ }^{2}$ Vascular Unit, Don Gnocchi Foundation, Milan, Italy

${ }^{3}$ Vascular Surgery and Angiology, University of Milan, Italy

A 1988 article in Chest questioned the possibility that patients with Multiple Sclerosis (MS) could develop Venous Thromboembolism (VTE).

Debilitation, reduced physical activity and hypomobility are present in several neurological diseases, and already at the time a correlation had been described between Deep Vein Thrombosis (DVT) and Pulmonary Embolism (PE) and sequelae of stroke and quadriplegia, but not for demyelinating diseases. The authors analysed data relating to 228 Patients suffering from MS, for a total of 1,986 MS-related hospitalisations over a period of 3 and a half years, and compared them with data relating to a total of 57,416 hospitalisations during the same period not due to MS. In no case were patients with MS hospitalised for VTE, while there were 433 in the control group [1].

Doctors working with and treating these patients reported episodic events of thromboembolism, at times even fatal, which took place either at home or during periods of hospitalisation, but until that point there had been no evidence that could confirm or disprove these claims.

Few years later, in 2010, our group published the data of a study conducted on MS patients hospitalised between January 2006 and December 2007 in a rehabilitation facility due to disabilities resulting from the disease [2], who were all subject to a compression ultrasound (CUS) during hospitalisation in order to detect signs of DVT in progress or sequelae of DVT.

The eligibility criteria, besides informed consent, included an assessment of the Kurtzke Scale or of the Expanded Disability Status Scale, and the ineligibility criteria included having recently taken potentially prothrombotic drugs or having taken anticoagulants for treatment or prevention. Blood samples were also taken from all Patients for a plasmatic D-dimer determination within 48 hours of hospitalisation.

A total of 132 patients were enrolled, and at the CUS 58 (43.9\%) showed signs of acute DVT ( ${ }^{\circ} 16$ ) or of previous DVT (no. 42 ). $55 \%$ reported a known history of VTE, and $60 \%$ suffered from chronic dependent edema. The D-dimer values were significantly higher in the group with acute or previous VTE $(594 \pm 657 \mathrm{ng} / \mathrm{dl}$ vs. $254 \pm 140 \mathrm{ng} / \mathrm{dl})$.

Except for those cases in which a recent onset of DVT could be assumed, it was not possible to determine the date of origin of the complication with certainty. This also made it difficult to decide which type of prophylaxis would be best since, inevitably, this could not be envisaged only during hospitalisation for any reason, but would also be needed for indefinite periods at home.

Such long-term pharmacological prophylaxis could have exposed Patients to complications, which could also prove harmful. Therefore, we focused on evaluating whether a mechanical prophylaxis with antiembolism stockings could be effective.

To answer this question, between April 2007 and September 2009 we conducted a new study in the same rehabilitation facility as the last, during which blood samples for a D-dimer determination were taken from 201 MS patients hospitalised for a period of motor rehabilitation lasting approximately 20 days. The samples were taken on admission and before discharge and the patients were examined with CUS within 48 hours of hospitalisation to rule out acute DVT, as well as cases of suspected DVT that may have emerged during hospitalisation. The first 99 were considered the "historical control group" and the next 102 the experimental group, who were made to wear knee-high anti-embolism stockings 24 hours a day.

None of the patients enrolled developed DVT during the brief hospitalisation. The patients treated with anti-embolism stockings managed dependent edema better and had significantly lower D-dimer values at discharge than the control group ( $24 \%$ vs. $7 \%$ of reduction in respect to the basal values) [3]

Christensen et al. recently published data extracted from the registers of Danish hospitalisations and compared hospitalised MS patients with the general population. Although the risk of developing VTE was not high, it was approximately three times higher in patients with MS compared to the control population, with a higher incidence in the first year after diagnosis [4].

The results of the MEGA study [5] conducted in 7 anticoagulation clinics in the Netherlands by collecting the data of 4311 Patients compared with 5768 control patients confirm these assumptions, demonstrating an odds ratio of 2.5 for VTE for MS patients with an absolute risk of $<4.6 \times 1000$ people per year, which in their opinion does not justify long-term pharmacological prophylaxis.

In conclusion, the data available up to this point seem to confirm a greater risk of VTE in Patients suffering from MS, but not enough to justify indefinite pharmacological prophylaxis. Our observations have shown in any case that there is a considerable prevalence of DVT and thrombosis sequelae in the patients studied. It is currently not possible to establish when a thrombotic complication is more likely to occur in the course of the disease. There seems to be a relationship with the presence of dependent edema, the result of greater hypomobility and damage to the skeletal-muscle pump, also demonstrated by higher D-dimer levels found in these patients.

Mechanical prophylaxis with anti-embolism stockings could perhaps find a role also due to the reduced risks of complications associated with them if properly used, but targeted studies on an adequate population for the proper period of time still must be conducted.

*Corresponding author: Arpaia G, Vascular Medicine, Desio and Vimercate General Hospital, Italy, Tel: +39 3356621211; E-mail: guido.arpaia@tin.it

Received December 19, 2013; Accepted January 24, 2014; Published February 05, 2014

Citation: Arpaia G, Bavera P, Ippolito E, Spezzigu G, Agus GB, et al. (2014) Risk of Thromboembolism in Patients Suffering from Multiple Sclerosis. J Neurol Neurophysiol S12: 002. doi:10.4172/2155-9562.S12-002

Copyright: @ 2014 Arpaia G, et al. This is an open-access article distributed unde the terms of the Creative Commons Attribution License, which permits unrestricted use, distribution, and reproduction in any medium, provided the original author and source are credited. 
Citation: Arpaia G, Bavera P, Ippolito E, Spezzigu G, Agus GB, et al. (2014) Risk of Thromboembolism in Patients Suffering from Multiple Sclerosis. J Neurol Neurophysiol S12: 002. doi:10.4172/2155-9562.S12-002

We Thanks CIZETA Medicali, Cuggiono, Italy for their unconditioned support.

\section{References}

1. Kaufman J, Khatri BO, Riendl P (1988) Are patients with multiple sclerosis protected from thrombophlebitis and pulmonary embolism? Chest 94: 9981001.

2. Arpaia G, Bavera PM, Caputo D, Mendozzi L, Cavarretta R, et al. (2010) Risk of deep venous thrombosis (DVT) in bedridden or wheelchair-bound multiple sclerosis patients: a prospective study. Thromb Res 125: 315-317.
3. Arpaia G, Bavera PM, Caputo D, Mendozzi L, Cavarretta R, et al. (2011) Effects of elastic compression on hypomobility edema and fibrinolysis activation in multiple sclerosis. Panminerva Med 53: 71-74.

4. Christensen S, Farkas DK, Pedersen L, Miret M, Christiansen CF, et al. (2012) Multiple sclerosis and risk of venous thromboembolism: a population-based cohort study. Neuroepidemiology 38: 76-83.

5. Ocak G, Vossen CY, Verduijn M, Dekker FW, Rosendaal FR, et al. (2013) Risk of venous thrombosis in patients with major illnesses: results from the MEGA study. J Thromb Haemost 11: 116-123. 